\title{
Human endometrial cell coculture reduces the endocrine disruptor toxicity on mouse embryo development
}

\author{
Myeong-Seop Lee ${ }^{2}$, Young-Sang Lee ${ }^{3}$, Hae-Hyeog Lee ${ }^{4}$ and Ho-Yeon Song ${ }^{1 *}$
}

\begin{abstract}
Backgrounds: Previous studies suggested that endocrine disruptors (ED) are toxic on preimplantation embryos and inhibit development of embryos in vitro culture. However, information about the toxicity of endocrine disruptors on preimplantation development of embryo in human reproductive environment is lacking.

Methods: Bisphenol A (BPA) and Aroclor 1254 (polychlorinated biphenyls) were used as endocrine disruptors in this study. Mouse 2-cell embryos were cultured in medium alone or vehicle or co-cultured with human endometrial epithelial layers in increasing ED concentrations.

Results: At 72 hours the percentage of normal blastocyst were decreased by ED in a dose-dependent manner while the co-culture system significantly enhanced the rate and reduced the toxicity of endocrine disruptors on the embryonic development in vitro.

Conclusions: In conclusion, although EDs have the toxic effect on embryo development, the co-culture with human endometrial cell reduced the preimplantation embryo from it thereby making human reproductive environment protective to preimplantation embryo from the toxicity of endocrine disruptors.
\end{abstract}

Keywords: Bisphenol A, Aroclor 1254, Mouse embryo, Human endometrial cells, Endocrine disruptors

\section{Backgrounds}

Endocrine disruptors are man made chemicals that have been shown to affect reproduction in wild life and may have adverse effects on humans [1-3]. Bisphenol A (BPA) is an important monomer used in the manufacture of a large number of chemicals and products, including epoxy and polystyrene resins that are used frequently in the food-packaging industry and in dentistry. Polychlorinated biphenyls (PCBs), a family of 209 highly stable synthetic congeners, were used for a decade in a wide range of industrial applications, due to their excellent flame retardant, lubricant, and electrical insulating properties.

Human exposure to these chemicals is significant. BPA have been detected in liquid from canned vegetables [4] and in the saliva of patients treated with dental sealants

\footnotetext{
* Correspondence: songmic@sch.ac.kr

'Department of Microbiology, School of Medicine, Soonchunhyang

University, Cheonan 330-090, South Korea

Full list of author information is available at the end of the article
}

[5]. The wide spread use and high chemical stability make PCBs ubiquitous and persistent in nature and their high lipophilicity causes these compounds to bio-accumulate, with the food chain being the main route of exposure. Especially, embryos, fetuses and newborns are highly susceptible to the exposure of these chemicals, which highlights the developmental toxicity of the compounds [6,7]. Further examples of exposure of human endometrium to the endocrine disruptors was demonstrated elsewhere although the levels were negligible [8]. Individual congener and total PCB concentrations were determined in serum (819-2627 pg/g whole weight) and follicular fluid (303-1257 pg/g whole weight) obtained from women undergoing assisted reproductive technologies (in vitro fertilization and embryo replacement) [9].

Endometrium is the main target for estrogens besides the breast, the pituitary, and the hypothalamus, and thus endometrium could be a useful tissue for evaluating the relevance of environmental estrogen exposure. Endometrial tissue is vital to important biological processes such as 
implantation and embryonic to blastocyst development, and disturbances in the function of this organ can lead to reduced fertility [8].

Embryos might be more sensitive to the endocrine disruptors than fetuses because preimplantation exposure might cause direct contact of endocrine disruptors that had entered maternal tissues, without placental barriers [10]. However, there remains a lack of knowledges about development of preimplantation embryos at the earliest stages in the presence of endocrine disruptors on human endometrium. So far, monolayers of purified epithelial cells or polarized epithelial cells have been used to investigate the effects of various materials on embryo development $[11,12]$. In a recent study, it was found that BPA partly affects implantation sites and alter the expression of implantation-associated genes [13]. Berger RG et. al. also showed that BPA exposure disrupts intrauterine implantation during early gestation [14]. However, the effects of BPA or BCP on the development of mouse embryos in presence or absence of human endrometrium epithelial cell have not been evaluated which can be interesting to understand the protective effect of embryos in cohort of co-culture system in vivo. In this study, we investigated the effects of two known endocrine disruptors, BPA or Aroclor 1254 (a commercial PCB; contains biphenyls with approximately $54 \%$ chlorine) on the development of mouse embryos (2-cells) co-cultured on human endometrial epithelial cell (hEEC) monolayers as an in vivo model by examination of dose dependency on embryo development.

\section{Methods}

\section{Human endometrial epithelial (hEEC) cell culture}

To study the development of mouse embryos under toxic exposure and their protective measures in vitro, toxic endocrine disruptors such as BPA or Arocolor1245 were used on mouse embryos. Therefore, this study was first approved by the Institutional Review Board
(IRB) of Soonchunhyang University Hospital, South Korea (IRB no. SCH 2009-36).

Human endometrial tissues were collected from normal cycling patients of the proliferative stages (days 414) who had undergone hysterectomy for uterine myoma at the Department of Obstetrics and Gynecology, Soonchunhyang University Hospital, from April 1 to July 30, 2009. For isolation and primary culture of human endometrial epithelial cells (hEECs), endometrial samples were processed following the method described by Ryan et al. [15] with minor modifications. Briefly, minced endometrial tissue was digested with collagenase (2 $\mathrm{mg} / \mathrm{ml}$; Gibco BRL, Grand Island, $\mathrm{NY}$ ) at $37^{\circ} \mathrm{C}$ in $\mathrm{Ca}^{++}, \mathrm{Mg}^{++}$-free PBS (Gibco, Carlsbud, CA, USA) for about $1 \mathrm{hr}$. Further mechanical separation was performed by repeated suction by pipetting. The suspension was serially filtered through a $100-\mu \mathrm{m}$ cell strainer (Falcon, Franklin Lakes, NJ, USA) and a $40-\mu \mathrm{m}$ cell strainer (Falcon). hEECs were backwashed from the $40-\mu \mathrm{m}$ cell strainer with the PBS and freed of collagenase by centrifugation (600 g, $5 \mathrm{~min}$ ). The cell pellet was resuspended in phenol red-free M199 (Gibco) containing antibiotics that was supplemented with $10 \%$ charcoal-dextran-treated fetal bovine serum (Hyclone Laboratories, South Logan, UT), 1nM E $\mathrm{E}_{2}$ (Sigma-Aldrich, St. Louis, MO, USA) and Insulin-Transferrin Sodium selenite ( $5 \mu \mathrm{g} / \mathrm{ml}$; Sigma-Aldrich). This medium was also used for embryo culture. Cell density was determined in a Neubauer hemocytometer. The cells were seeded on 4-well dishes and cultured until confluency at $37^{\circ} \mathrm{C}$ in a humidified atmosphere of $5 \% \mathrm{CO}_{2}$ in air. The epithelial cells later grew as whorl around the explants (Figure 1) and cell viability was greater than $90 \%$. After reaching $80 \%$ confluence in medium cells were found polyhedral in shape and epithelioid characteristics of epithelial cells. The homogeneity of cultures was determined by morphologic characteristics and verified by immunocytochemical localization of cytokeratin, vimentin, as described elsewhere

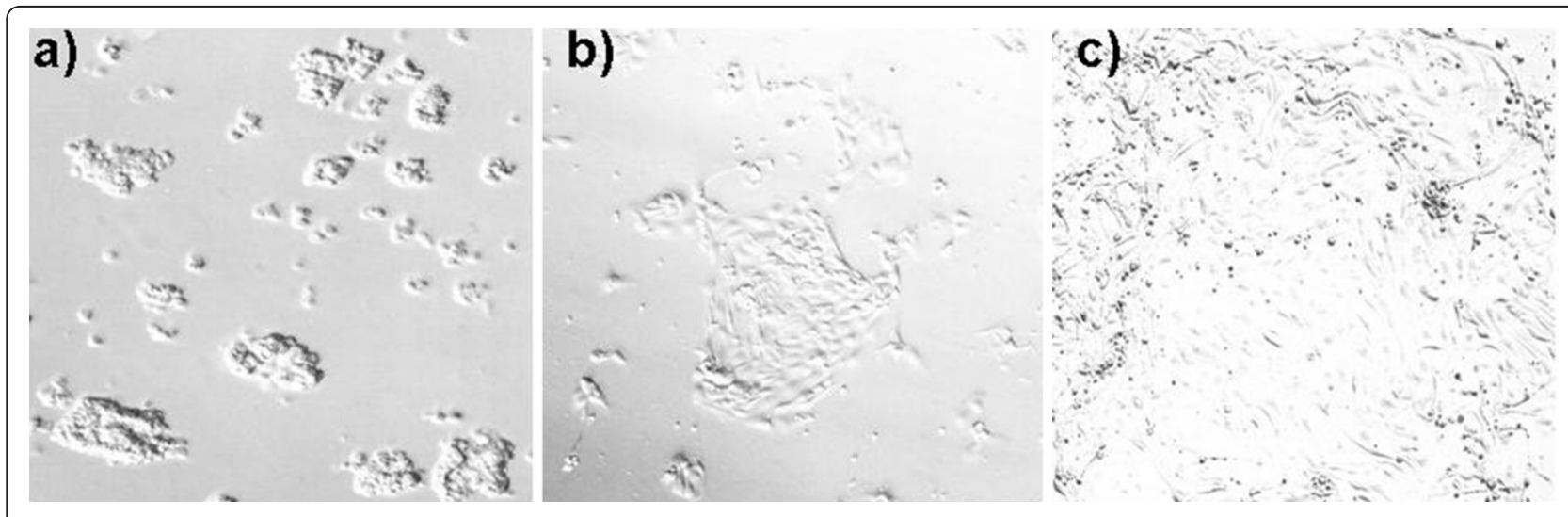

Figure 1 Morphology of human endometrial epithelial cells isolated freshly (a), attached (b) and confluent (c) (X100). 
[16]. After primary culture (day 4) cells were examined. Antiserum to cytokeratin reacted strongly with EEC and revealed the characteristic cytoarchitecture showing intermediate filamentous orientation of cytokeratins.

\section{Retrieval of mouse 2-cell embryos and Endocrine disruptor treatments}

A total of 149 mice were used in this study, 75 of which for PCB and 74 for BCP. Five-week-old ICR female mice (from Korea Research Institute of Bioscience and Biotechnology, Daejeon, Korea) were injected with 10 IU of intraperitoneal pregnant mare serum gonadotropin and with $10 \mathrm{IU}$ intraperitoneal human chorionic gonadotrophin (hCG) $48 \mathrm{hrs}$ later. They were impregnated overnight by 12 -week-old males of the same strain. Mating was ascertained by the appearance of a vaginal plug on the following morning. Female mice were killed at $48 \mathrm{hr}$ after hCG administration by cervical dislocation and mouse 2-cell embryos were flushed from the oviducts.

Mouse 2-cell embryos were cultured in the medium for $72 \mathrm{hrs}$ in increasing BPA (Sigma-Aldrich) concentrations $\left(0,10^{-8}, 10^{-6}, 10^{-4} \mathrm{M}\right)$ or Aroclor $1254(200 \mu \mathrm{g} / \mathrm{ml}$ dissolved in methanol; Supelco) concentrations (0, 0.02, $0.2,1 \mu \mathrm{g} / \mathrm{ml}$ ) to investigate the dose dependent toxicities of BPA and Aroclor 1254 on the embryonic development. The concentration of $0 \mathrm{M}(\mathrm{BPA})$ or $0 \mu \mathrm{g} / \mathrm{ml}$ (Aroclor 1254) was control group. The percentages of blastocyst were determined as the rate of development of blastocyst at 72 hour (Figures 2, 3). The $E_{2}$ and BPA were dissolved in ethanol as stock solutions and Aroclor 1254 in methanol. The concentration of vehicle solution added into the culture medium was $0.01 \%$ (ethanol) or $0.05 \%$ (methanol), respectively. Co-culture was done with the hEEC monolayer in the medium as described above. Blastocysts were stained with Hoechst 33342 and the number of cells in the blastocysts was counted to determine whether degenerated or not (Figure 4).

\section{Statistical analysis}

Ki square test was performed on the data in order to determine significant differences $(\mathrm{P}<0.05)$ between the treatment (media contains a series of increasing concentrations of endocrine disruptors) and control (not treated with any of the endocrine disruptors; i.e. - media contains null/zero concentration of endocrine disruptors) groups.

\section{Results}

To investigate the toxicological impact of endocrine disruptors (BPA or Aroclor 1254) and the effect of human reproductive environment, dose dependent concentrations of the endocrine disruptors were exposed on mouse 2-cell embryos cultured in the medium or on hEEC monolayers and the embryonic development was compared to that of control (media without any endocrine disruptors). Tables 1 and 2 showed the dose dependent toxicities of BPA and Aroclor 1254 on the preimplantation development of embryos and the protective effect of human reproductive environment on preimplantation embryos.

\section{Effect of BPA}

From Table 1 it was clear that BPA at $10^{-4} \mathrm{M}$ concentrations had the greatest detrimental effect on the development of embryos at 72 hour of culture. No blastocyst was observed in case of both medium alone and vehicle and only $50 \%$ blastocysts were observed for coculture system $(\mathrm{p}<0.05)$. It was also evident that when the concentration of BPA was increased, the total number of a)

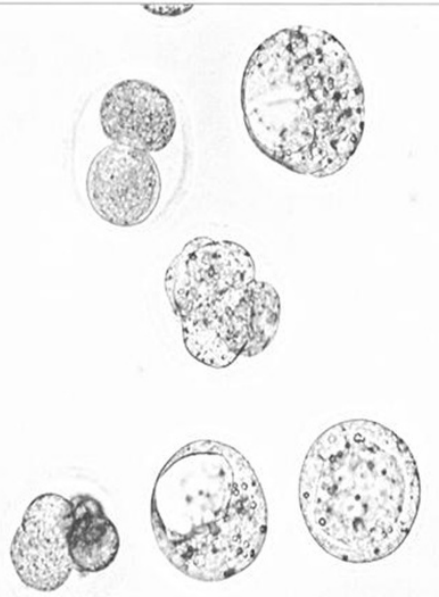

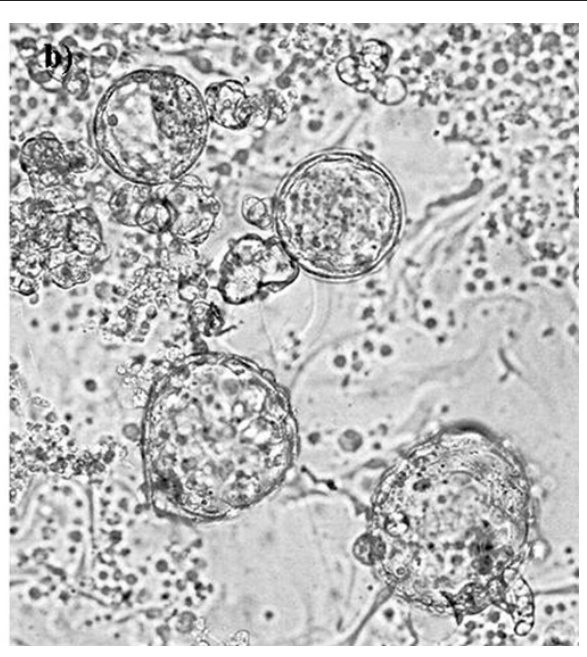

Figure 2 Morphology of mouse embryos developed normally in the medium (a) or the coculture (b) (X100). 


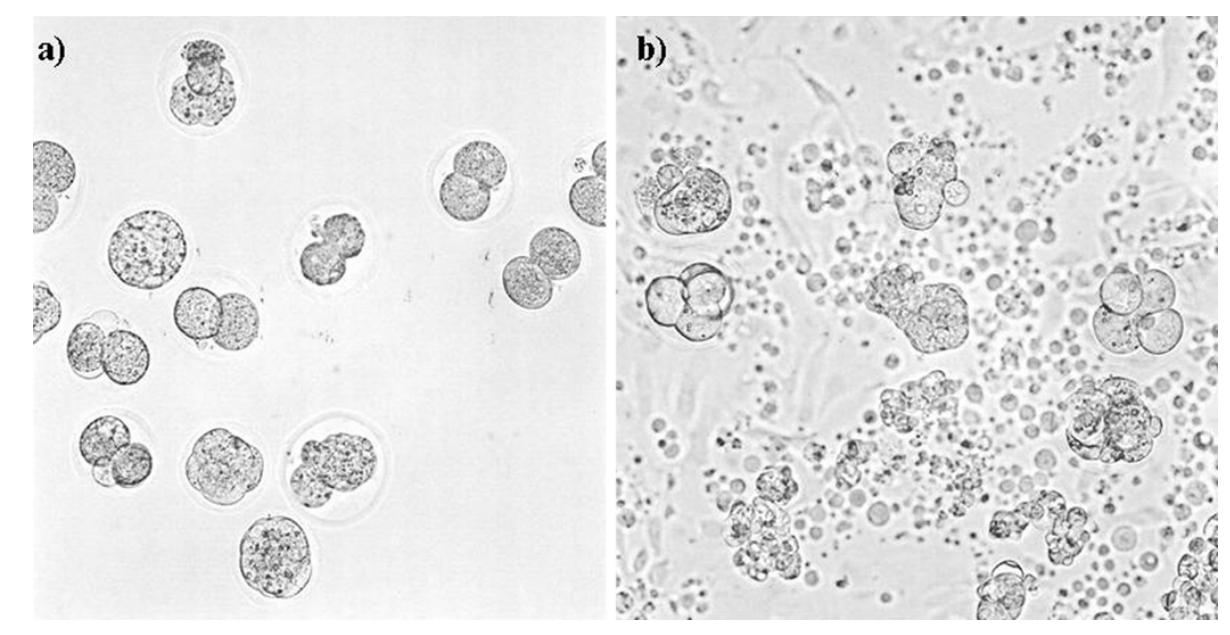

Figure 3 Morphology of mouse embryos degenerated by endocrine disruptors in the medium (a) or the coculture (b) (X100).

blastocysts was also decreased. However, coculture with hEEC monolayers was found to be beneficial because the decreasing rate of blastocysts number was much smaller than that of the medium or vehicle (Table 1).

\section{Effect of Aroclor 1254}

Similar dose dependent effect of Aroclor 1254 at different concentrations was observed on embryonic development in Table 2. The detrimental effect of Aroclor 1254 on embryonic development was found to be increasing with the increase of concentration in all treatment groups at 72 hour. Especially, $1 \mu \mathrm{g} / \mathrm{ml}$ Aroclor 1254 showed the most detrimental effect. In this concentration only $43 \%$ blastocysts were observed for co-culture system, but none for media alone and vehicle $(\mathrm{P}<0.05)$. Also in this case, co- culture was found beneficial by decreasing the rate of detrimental effect Aroclor 1254 on embryonic development (Table 2). Thus these results showed that the hEEC coculture alleviated the toxicity of BPA or Aroclor 1254 on the embryonic development, especially in the high dose.

\section{Discussion}

Three different types of cells, e.g. - the epithelium, the endometrial stroma and the myometrium, are known to found in mammalian uterus. The differentiation of endometrial epithelia is considered important regarding successful embryonic development, placentation or embryo implantation. But due to environmental pollution and extensive distribution of PCBs in the ecosystem, endometrial cells have over exposed to these carcinogenic
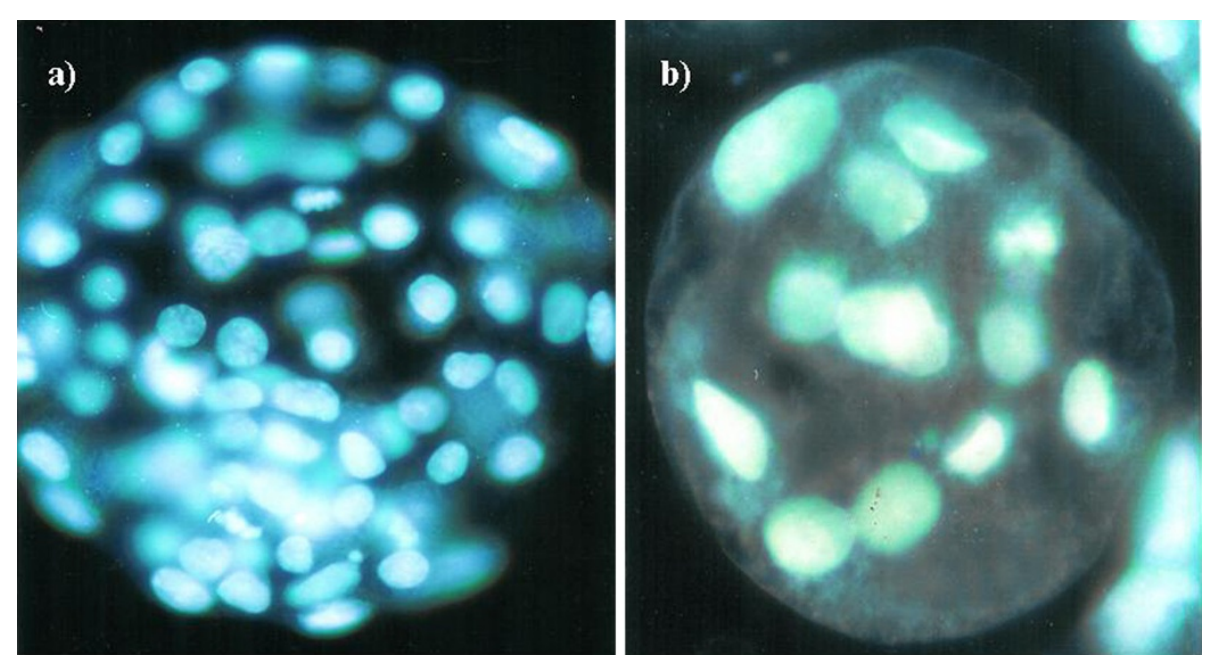

Figure 4 Fluorescence micrograph of normal (a) and abnormal (b) mouse blastocyst stained with Hoechst 33342 (X400). 
Table 1 The effect of coculture on development of mouse embryos in increasing Bisphenol A

\begin{tabular}{lcccc}
\hline $\begin{array}{c}\text { Concentrations } \\
(\mathbf{M})\end{array}$ & $\begin{array}{c}\text { Embryos } \\
\text { grown on }^{*}\end{array}$ & $\begin{array}{c}\text { Total } \\
\text { number of } \\
\text { embryos }\end{array}$ & $\begin{array}{c}\text { Number of } \\
\text { blastocysts }\end{array}$ & $\begin{array}{c}\text { \% of } \\
\text { blastocyst }\end{array}$ \\
\hline 0 & Medium alone & 125 & 116 & 93 \\
& Vehicle & 144 & 130 & 90 \\
$10^{-8}$ & Coculture & 112 & 110 & $98^{\mathrm{b}}$ \\
& Medium alone & 121 & 102 & 84 \\
$10^{-6}$ & Vehicle & 132 & 107 & 81 \\
& Coculture & 123 & 111 & $90^{\mathrm{b}}$ \\
& Medium alone & 120 & 80 & 67 \\
$10^{-4}$ & Vehicle & 123 & 76 & 62 \\
& Coculture & 121 & 87 & $72^{\mathrm{b}}$ \\
& Medium alone & 135 & 0 & $0^{\mathrm{c}}$ \\
& Vehicle & 113 & 0 & $0^{\mathrm{c}}$ \\
& Coculture & 123 & 62 & $50^{\mathrm{bc}}$ \\
\hline
\end{tabular}

*Mouse embryos were grown on three different kinds of culture system. In Medium alone M199 (Gibco) media were used. In Vehicle, vehicle solutions (ethanol/methanol) were used. In co-culture, embryos were grown on human endometrial epithelial cells ( $\mathrm{hEECs}$ ).

aThe percentages of blastocyst at 72 hour of culture.

${ }^{b}$ Values in coculture were significantly higher than those in medium alone, respectively: $P<0.05$.

'Values were significantly lower than those of each control $(0 \mu \mathrm{g} / \mathrm{ml})$, respectively: $P<0.05$.

chemicals and several studies have reported the detrimental effects PCBs on animal reproduction systems. In human beings, complications such as reduced birth weight, diminished head circumference and reduced gestational age have been reported to occur due to maternal consumption of PCB contaminated fish $[17,18]$. Among various chemical formulations, BPA and Aroclor 1254 are the known endocrine disruptors of the PCB family.

To date, several studies have indicated that BPA mimics estrogen and $10^{-8}$ to $10^{-6} \mathrm{M}$ BPA exerts estrogenic effects on various kind of cells in vitro $[10,14,19]$ and over 10 ${ }^{-5} \mathrm{M}$ BPA is cytotoxic [20]. On the other hand, one to $10 \mu \mathrm{g} / \mathrm{ml}$ Aroclor 1254 was found cytotoxic [21-23].

In this study, endocrine disruptors decreased the developmental rates of embryos by dose dependency (Table 1,2). Takai et al [10]. have shown that BPA significantly decreased the developmental rate of two-cell mouse embryos to blastocysts in vitro. Kholkute et al. $[21,22]$ examined the effect of Aroclor 1254 on the development of 2-cell embryos to the 4-cell stage at $48 \mathrm{hr}$. They found that increasing the concentration of Aroclor 1254 in the culture medium significantly reduced the progression of 2-cell embryos to the 4-cell stage or greater at $48 \mathrm{hr}$. Also, the presence of Aroclor 1254 reduced both the in vitro fertilization rates and embryonic development in mice. The results demonstrated that increasing concentrations of BPA or Aroclor 1254 had a deleterious effect directly on embryonic development.
During the blastulation, differentiation events were occurred and contact-induced cell polarization and reorganization of cytoskeletal elements were expressed. In this study, the blastulation at $72 \mathrm{hr}$ in the medium alone and vehicle was not observed at $10^{-4} \mathrm{M}$ (BPA) or $1 \mu \mathrm{g} / \mathrm{ml}$ (Aroclor 1254). It might be due to the fact that the chemicals have aneuploidogenic properties that inhibit MT assembly in intact cells. Pfeiffer et al. [24] demonstrated by staining with anti-centromere antibodies that all micronuclei in V79 cells induced by BPA contained whole chromatids/chromosomes, suggesting that BPA does not induce DNA breakage. Tsutsui et al. [25] reported that BPA exerted transforming and genotoxic effects on cultured syrian hamster embryo (SHE) cells and the DNA adduct formation observed in cultured SHE cells treated with BPA was involved in nondisjunction, leading to aneuploidy. Takai et al. [26] demonstrated that embryos that did not reach the blastocyst stage at $48 \mathrm{hrs}$ were largely in the morula stage, although the percentage of degenerated embryos was significantly increased in the $10^{-4} \mathrm{M}$ BPA-exposed group. Hernandez and Dukelow reported that the morula was more susceptible to the toxic effect of Aroclor 1254 and Aroclor 1254 could affect the development of the embryos by reducing the number of gap junctions [23]. Several other studies indicate that even human endometrial cells are susceptible to the exposure of ED

Table 2 The effect of coculture on development of mouse embryos in increasing Aroclor 1254 concentrations

\begin{tabular}{|c|c|c|c|c|}
\hline $\begin{array}{l}\text { Concentrations } \\
(\mu \mathrm{g} / \mathrm{ml}) \mathrm{fs}\end{array}$ & Group & $\begin{array}{c}\text { Total } \\
\text { number of } \\
\text { embryos }\end{array}$ & $\begin{array}{c}\text { Number } \\
\text { of } \\
\text { blastocysts }\end{array}$ & $\begin{array}{c}\text { \% of } \\
\text { blastocyst }^{a}\end{array}$ \\
\hline \multirow[t]{3}{*}{0} & Medium alone & 122 & 111 & 91 \\
\hline & Vehicle & 113 & 102 & 90 \\
\hline & Coculture & 133 & 125 & $94^{b}$ \\
\hline \multirow[t]{3}{*}{0.02} & Medium alone & 133 & 109 & 82 \\
\hline & Vehicle & 121 & 97 & 80 \\
\hline & Coculture & 125 & 110 & $88^{b}$ \\
\hline \multirow[t]{3}{*}{0.2} & Medium alone & 121 & 76 & 63 \\
\hline & Vehicle & 115 & 69 & 60 \\
\hline & Coculture & 120 & 82 & $68^{b}$ \\
\hline \multirow[t]{3}{*}{1} & Medium alone & 122 & 0 & $0^{c}$ \\
\hline & Vehicle & 119 & 0 & $0^{c}$ \\
\hline & Coculture & 128 & 55 & $43^{b c}$ \\
\hline
\end{tabular}

*Mouse embryos were grown on three different kinds of culture system. In Medium alone M199 (Gibco) media were used. In Vehicle, vehicle solutions (ethanol/methanol) were used. In co-culture, embryos were grown on human endometrial epithelial cells ( $\mathrm{hEECs}$ ).

${ }^{\mathrm{a}}$ The percentages of blastocyst at 72 hour of culture.

${ }^{b}$ Values in coculture were significantly higher than those in medium alone, respectively: $P<0.05$.

'Values were significantly lower than those of each control $(0 \mu \mathrm{g} / \mathrm{ml})$, respectively: $P<0.05$. 
[27,28]. In this study, BPL and Aroclor 1254 might have some toxic effect on hEEC too, but co-culturing mouse embryos with hEEC monolayer cells is advantageous.

The beneficial effects of co-culture include the secretion of embryotrophic factors such as nutrients and substrates, growth factors and cytokines and the removal of potentially harmful substances such as heavy metals, ammonium, and free radical formation, detoxifying the culture medium [29]. In this study, the co-culture with hEECs increased the percentages of blastocyst in all treatments. However, it should be noted that the total cell in co-culture system might be higher than that of the medium alone or vehicle. Therefore, decreasing the BPA or Arocolor-1245 concentration in the co-culture system might have a reduced toxic effect as compared with medium alone or vehicle culturing. In other cases, bio-transformation of these chemicals causes bio-accumulation in increased concentrations.

Because of ethical and legal issues, an in vitro model was used to elucidate the impacts of endocrine disruptors on embryonic development. The results should be considered in light of the limitations of an in vitro model. In addition, mouse embryos were used rather than human embryos. Nevertheless, the results from this study were useful in evaluating impacts of endocrine disruptors at the human endometrial-embryonic interface.

\section{Conclusions}

In conclusion, increasing concentrations of BPA or Aroclor 1254 had a deleterious effect directly on embryonic development. This suggests that the endocrine disruptors, BPA and Aroclor 1254 might dose dependently reduce the embryo development on reproductive environment. However, in this study, we showed mouse endometrial cell coculture reduced the toxicities of BPA and Aroclor 1254 on preimplantation development of embryos and this suggests that reproductive environment protects preimplantation embryo from the toxicity of endocrine disruptor.

\section{Competing interests}

The authors report no competing interests with any person or institution.

\section{Authors' contributions}

$M-S L$ and $H-Y S$ provided the idea for the study. M-S L, $Y-S L, H-H L$ did in vitro works; $M-S L, Y-S L, H-H L$ and $Y-S L$ analyzed and interpreted the data. M-S L and H-Y S wrote the paper. All authors revised it and approved the version to be published. $\mathrm{H}-\mathrm{Y} \mathrm{S}$ is the guarantor. All authors read and approved the final manuscript.

\section{Author details}

${ }^{1}$ Department of Microbiology, School of Medicine, Soonchunhyang University, Cheonan 330-090, South Korea. ${ }^{2}$ Industry Academy Cooperation Foundation, Soonchunhyang University, Asan, 336-745, South Korea. ${ }^{3}$ Department of Biomedical Technolgy, Soonchunhyang University, Asan, 336-745, South Korea. ${ }^{4}$ Department of Obstetrics and Gynecology, Soonchunhyang University Bucheon Hospital, Bucheon, 420-767, South Korea.
Received: 9 March 2011 Accepted: 14 March 2012

Published: 30 April 2012

\section{References}

1. Colborn T, vom Saal FS, Soto AM: Developmental effects of endocrine-disrupting chemicals in wildlife and humans. Environ Health Perspect 1993, 101:378-384.

2. Daston GP, et al: Environmental estrogen and reproductive health: a discussion of the human and environmental data. Reprod Toxicol 1997 11:465-481

3. Sonnenschein C, Soto AM: An updated review of environmental estrogen and androgen mimics and antagonists. J Steroid Biochem Mol Biol 1998, 65:143-150

4. Brotons JA, et al: Xenoestrogens released from lacquer coatings in food cans. Environ Health Perspect 1995, 103:608-612.

5. Olea $\mathrm{N}$, et al: Estrogenicity of resin-base composites and sealants used in dentistry. Environ Health Perspect 1996, 104:298-305.

6. Kim JC, et al: Evaluation of developmental toxicity in rats exposed to the environmental estrogen bisphenol A during pregnancy. Life Sci 2001, 69:2611-2625.

7. Coletti D, et al: Polychlorobiphenys inhibit skeletal muscle differentiation in culture. Toxicol Appl Pharmacol 2001, 175:226-233.

8. Schaefer WR, et al: Exposure of human endometrium to environmental estrogens, antiandrogens, and organochlorine compounds. Fertil Steril 2000, 74:558-563.

9. Pauwels $A$, et al: The relation between levels of selected PCB congeners in human serum and follicular fluid. Chemosphere 1999, 39:2433-2441.

10. Takai $Y$, et al: Estrogen receptor-mediated effects of a xenoestrogen, bisphenol A, on preimplantation mouse embryos. Biochem Biophys Res Commun 2000, 270:918-921.

11. Carrasco I, Cebral E, Benitez R, Vantman D: Hydrosalpinx fluid affects murine embryonic development in a coculture system with epithelial endometrial cells. Fertil Steril 2001, 75:1004-1008.

12. Valbuena $D$, et al: Increasing levels of estradiol are deterious to embryonic implantation because they directly affect the embryo. Fertil Steril 2001, 76:962-968.

13. Varayoud J, Ramos JG, Bosquiazzo VL, Lower M, Munoz-de-Toro M, Luque EH: Neonatal exposure to bisphenol A alters rat uterine implantationassociated gene expression and reduces the number of implantation sites. Endocrinology 2011, 152(3):1101-1111.

14. Berger RG, Foster WG, de Catanzaro D: Bisphenol-A exposure during the period of blastocyst implantation alters uterine morphology and perturbs measures of estrogen and progesterone receptor expression in mice. Reprod Toxicol 2010, 30(3):393-400.

15. Ryan IP, Schriock ED, Taylor RN: Isolation, characterization, and comparison of human endometrial and endometriosis cells in vitro. $J$ Clin Endocrinol Metab 1994, 78:642-649.

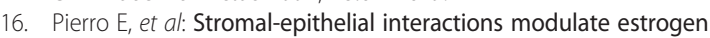
responsiveness in normal human endometrium. Biol Reprod 2001, 64:831-838

17. Fein GG, et al: Prenatal exposure to polychlorinated biphenyls: effects on birth size and gestational age. J Pediatr 1984, 105:315-320.

18. Swain WR: Effects of organochlorine chemicals on the reproductive outcome of humans who consumed contaminated great lake fish: an epidemiological consideration. J Toxicol Environ Health 1991, 33:587-639.

19. Krishnan $A$, et al: Bisphenol-A: an estrogenic substance is released from polycarbonate flasks during autoclaving. Endocrinology 1993, 132:2279-2286

20. Nakagawa $Y$, Tayama S: Metabolism and cytotoxicity of bisphenol A and other bisphenols in isolated rat hepatocytes. Arch Toxicol 2000, 74:99-105.

21. Kholkute SD, Rodriguez J, Dukelow WR: Reproductive toxicology of Aroclor-1254: effects on oocyte, spermatozoa, in vitro fertilization, and embryo development in the mouse. Reprod Toxicol 1994, 8:487-493.

22. Kholkute SD, Rodriguez J, Dukelow WR: Effects of polychlorinated biphenyls (PCBs) on in vitro fertilization in the mouse. Reprod Toxicol 1994, 8:69-73.

23. Hernandez O, Dukelow WR: Aroclor-1254 effects on the in vitro development of 8-cell mouse embryos. Bul Environ Contam Toxicol 1998, 60:773-780 
24. Pfeiffer E, Rosenberg B, Deuschel S, Metzler M: Interference with microtubules and induction of micronuclei in vitro by various bisphenols. Mut Res 1997, 390:21-31.

25. Tsutui T, et al: Bisphenol-A induces cellular transformation, aneuploidy and DNA adduct formation in cultured Syrian hamster embryo cells. Int J Cancer 1998, 75:290-294.

26. Takai Y, et al: Preimplantation exposure to bisphenol A advances postnatal development. Reprod Toxicol 2001, 15:71-74.

27. Aghajanova L, Giudice LC: Effect of bisphenol A on human endometrial stromal fibroblasts in vitro. Reprod Biomed Online 2011, 22(3):249-56. Epub 2010 Dec 23.

28. Bredhult C, Backlin BM: Olovsson M Effects of some endocrine disruptors on the proliferation and viability of human endometrial endothelial cells in vitro. Reprod Toxicol 2007, 23(4):550-559.

29. Bavister BD: Culture of preimplantation embryos: facts and artifacts. Hum Reprod Update 1995, 1:91-148.

doi:10.1186/1745-6673-7-7

Cite this article as: Lee et al.: Human endometrial cell coculture reduces the endocrine disruptor toxicity on mouse embryo development.

Journal of Occupational Medicine and Toxicology 2012 7:7.

\section{Submit your next manuscript to BioMed Central and take full advantage of:}

- Convenient online submission

- Thorough peer review

- No space constraints or color figure charges

- Immediate publication on acceptance

- Inclusion in PubMed, CAS, Scopus and Google Scholar

- Research which is freely available for redistribution 